\title{
Mit Feedback-Systemen führen
}

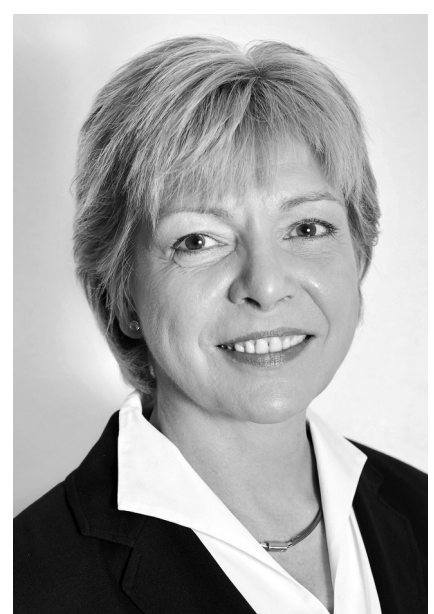

VON SABINE MACHWÜRTH Sabine Machwürth ist geschäftsführende Gesellschafterin der Unternehmensberatung Machwürth Team International (MTI Consultancy), für die weltweit 450 Berater, Trainer und Projektmanager tätig sind.

www.mticonsultancy.com

\author{
Kennzahlen und Feedback-Systeme machen den \\ Erfolg von Unternehmen und deren Entwicklung \\ transparent - sofern die gesammelten Daten \\ richtig interpretiert und genutzt werden. Das \\ setzt neben einer stimmigen Infrastruktur auch \\ einige Fähigkeiten der Führungskräfte voraus.
}

Zahlen sind ein bewährtes Mittel, um die Entwicklung von und in Unternehmen transparent zu machen und deren Erfolg zu steuern. Doch schon lange nicht mehr beschränkt sich das Messen und Transparent machen auf das klassische bilanzorientierte beziehungsweise betriebswirtschaftliche Controlling. Denn inzwischen haben viele erkannt, welch großen Einfluss die sogenannten weichen Faktoren wie zum Beispiel die Identifikation der Mitarbeiter mit dem Unternehmen und deren Arbeitszufriedenheit auf die harten Zahlen haben. Deshalb messen moderne ManagementInformationssysteme auch die "weichen Erfolgsfaktoren «, da sie letztlich zu den harten Zahlen führen.

\section{Daten erfassen und bewerten}

Für das Gewinnen der zum Steuern nötigen Daten und Informationen gibt es zahlreiche Instrumente. Hierzu zählen unter anderem Kunden- und Mitarbeiterbefragungen, Potenzialanalysen für Führungskräfte und Mitarbeiter, Mitarbeiter- und Vorgesetzten-Beurteilungen, Führungsstilanalysen, Team-Feedbacks sowie Förder- und Zielvereinbarungsgespräche. Zudem wird in vielen Unternehmen, um die Qualität und somit Leistung zu steigern, eine systematische Fehlersuche im Sinne des Kontinuierlichen Verbesserungsprozesses betrieben. Außerdem werden die nötigen Korrekturen und erzielten Verbesserungen mit System erfasst. Feedback-Instrumente zum Erfassung der weichen Faktoren sind u. a.:

- Kundenbefragungen

- Mitarbeiterbefragungen

- Klimaanalysen

- Führungsstilanalysen

- Potentialanalysen auf allen Ebenen

- Mitarbeiterbeurteilung

- Vorgesetztenbeurteilung

- Teamfeedback auf allen Ebenen

- Förder- und Zielvereinbarungsgespräche

Ein Merkmal dieser Messinstrumente ist: Sie liefern den Führungskräften in den Unternehmen zunächst nur eine Vielzahl von Daten und Informationen. Diese gilt es zu verarbeiten und bewerten, damit hieraus die erforderlichen Schlüsse gezogen werden können.

Eine zentrale Voraussetzung hierfür ist, dass ein Management-Informationssystem existiert, in dem die Daten systematisch erfasst und regelmäßig aktualisiert werden - und zwar so, dass sie leicht und komprimiert abrufbar sind. Das erfordert ein Übersetzen und Vereinheitlichen der gewonnenen Informationen, sodass das System mit wenigen Kennzahlen, sprich Messgrößen auskommt. Hierfür müssen die eingesetzten Feedback-Systeme aufeinander abgestimmt werden. Sonst besteht die Gefahr, dass sie den Führungskräften zwar eine Vielzahl von Daten liefern, die diese aber nicht bewerten und somit auch nicht für ihre Arbeit nutzen können. 


\section{Die Daten kommunizieren}

In vielen Unternehmen werden Zahlen (und Ergebnisse) traditionell als Führungssache und somit Geheimsache betrachtet: Die Führungskräfte allein sichten und interpretieren die Daten. Und anschließend leiten sie daraus ihre Beschlüsse $a b$ und erteilen ihren Untergebenen entsprechende Anweisungen.

Ein solches Arbeiten mit Kennzahlen ist verfehlt. Denn es schafft nur Transparenz für die Führungskräfte, während für ihre Mitarbeiter im Dunkeln bleibt, warum bestimmte Entscheidungen getroffen wurden und warum gewisse Maßnahmen nötig sind. Entsprechend gering ist ihr Engagement, und entsprechend abhängig bleiben sie bei ihrer Arbeit von den Weisungen ihrer Führungskräfte.

Für das Arbeiten mit Feedback-Systemen gilt: Das Optimum wird nur erreicht, wenn die Führungskräfte ihre Mitarbeiter teamorientiert führen. Das heißt, die Kennzahlen und Daten müssen dem Team transparent gemacht werden. Außerdem sollten, soweit möglich, mit dem Team die nötigen Schlüsse gezogen und die Entwicklungen in dem Bereich geplant werden. Deshalb sollte jede Führungskraft für ihren Bereich die erforderlichen Datenübersichten zusammenstellen (lassen).

\section{Aus Daten richtige Beschlüsse ableiten}

Dabei gilt es zwischen den Leistungsdaten, die primär Auskunft über das (betriebswirtschaftlich) Erreichte geben, und den weichen Daten, die unter anderem Auskunft über die (Entwicklung der) Kultur des Unternehmens geben, zu unterscheiden. Die Leistungsdaten sollten kontinuierlich erfasst und mindestens einmal pro Monat in einer Teamsitzung besprochen werden.

Eine Voraussetzung hierfür ist, dass die Ziele wirklich als Arbeitsziele quantifiziert sind, deren Erreichen messbar ist. Dasselbe gilt für die Daten, die Auskunft über die Fehler beziehungsweise Qualitätsmängel sowie über den Stand der zu ihrer Vermeidung ergriffenen Maßnahmen geben. Auch ihr Besprechen sollte eine feste Routine sein. Das heißt, zum Beispiel einmal pro Monat sollte in einer Teamsitzung über die aufgetretenen Mängel, die unter anderem zu Verschwendung oder einer geringen Kundenzufriedenheit führen, und den Stand der Maßnahmen zu ihrer Beseitigung gesprochen werden.

\section{Mit Kennzahlen führen in der Sozialwirtschaft}

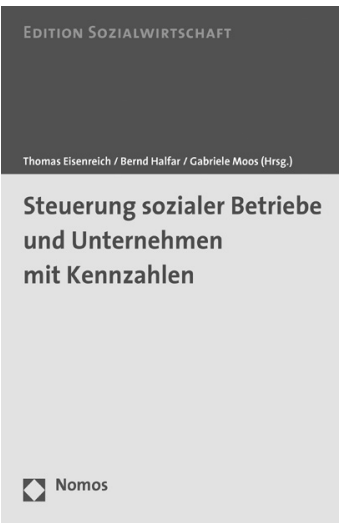

Kennzahlen sind das Cockpit des Managements. Inzwischen hat sich auch in sozialen Unternehmen die Erkenntnis durchgesetzt, dass Kennzahlen ein wichtiges Instrument sind, um den Unternehmenserfolg zu steuern. Die aus der gewerblichen Wirtschaft bekannten Kennzahlen sind als Werkzeuge für soziale Unternehmen nicht oder nur bedingt geeignet - eigene Kennzahlsysteme

Auch über die Entwicklung der weichen Faktoren sollten die Mitarbeiter regelmäßig - zum Beispiel im Quartalsrhythmus - informiert werden, um anschließend mit dem Team aus den Feedback-Ergebnissen (weitere) Entwicklungsmaßnahmen abzuleiten und deren Stand zu reflektieren und gegebenenfalls zu korrigieren. So sollten zum Beispiel die aus den Zielvereinbarungs- und Fördergesprächen abgeleiteten Maßnahmen für alle Mitarbeiter transparent sein.

Zudem empfiehlt es sich, einmal jährlich mit dem Team in einer Klausurtagung die Ergebnisse aus allen FeedbackInstrumenten des Unternehmens für den eigenen Bereich zu bewerten. Dann können anschließend aus den Ergebnissen die Maßnahmen für das Folgejahr und Schritte zu deren Realisierung abgeleitet werden. Festgelegt werden sollte bei diesem Meeting auch, in welchen Zeitabständen diese Daten mit der zwischenzeitlichen Entwicklung abgeglichen werden. Empfehlenswert ist eine quartalsmäßige Reflexion des Entwicklungsstands.

Um das Umsetzen der geplanten und vereinbarten Maßnahmen verfolgen zu können, ist es ratsam, die Aktivitäten mit Hilfe eines EDV-Tools zum Beispiel zur Verwaltung von Projekten systematisch zu erfassen. Hierbei sollte darauf geachtet werden, dass die Maßnahmen nach Handlungsfeldern und Verantwortlichen gelistet werden können; des sind zwingend notwendig. Das leider im Buchhandel vergriffene Buch "Steuerung sozialer Betriebe und Unternehmen mit Kennzahlen « ist in Bibliotheken und antiquarisch noch erhältlich. Es bereitet das komplexe Thema verständlich auf und stellt ein umfassendes Kennzahlenlexikon für die Steuerung von sozialen Unternehmen zur Verfügung. Das Werk erläutert die Bedeutung von Kennzahlen für soziale Unternehmen, macht den Nutzen von finanziellen und nicht-finanziellen Kennzahlen für die erfolgreiche Unternehmenssteuerung deutlich und verdeutlicht anhand von Praxisbeispielen aus sozialen Unternehmen, die Chancen und Risiken von Kennzahlen. Das Buch war als Band 22 in der Reihe Edition Sozialwirtschaft erschienen.

Thomas Eisenreich, Bernd Halfar, Gabriele Moos: Steuerung sozialer Betriebe und Unternehmen mit Kennzahlen. Nomos Verlagsgesellschaft, Baden-Baden 2004. 166 Seiten. 34,- Euro. ISBN 978-3-8329-0714-3.

Weiteren danach, welches Ziel mit ihnen erreicht werden soll.

\section{Anforderungen an die Führung}

Die Organisation der Daten und das teamorientierte Führen ihrer Mitarbeiter mit Hilfe der Daten stellen gewisse Anforderungen an die Führungskräfte. Daneben benötigen Führungskräfte, die die Arbeit und Entwicklung in ihrem Bereich mit Feedback-Systemen steuern möchten, auch gewisse strategische Fähigkeiten - unter anderem, weil sie hierbei die Zukunft gedanklich vorwegnehmen müssen. Außerdem müssen sie über die Kompetenz verfügen, Veränderungen vorzubereiten und zu initiieren.

Das Entwickeln der hierfür erforderlichen Fähigkeiten sollte ein fester Bestandteil der Führungskräfteentwicklung sein. Denn künftig müssen Führungskräfte nicht nur in der Lage sein, den Betriebsalltag zu managen. Sie müssen auch die Zukunft gestalten können. Hierfür benötigen sie die Fähigkeit, mit Hilfe von Feedback-Systemen, Signale, die Risiken, aber auch Chancen anzeigen, frühzeitig zu erkennen. Denn nur dann können sie aktiv werden und mit ihren Teams zum Beispiel Szenarien und Maßnahmen entwerfen, die sicherstellen, dass ihre Bereiche auch künftig TopLeistungen erbringen und ihre Funktion in der Organisation erfüllen. 